\title{
Flare-Ups in Endodontics - A Review
}

\author{
Priyanka.S.R , Dr.Veronica \\ (Saveetha Dental college, Saveetha University, India) \\ (Department of Conservative dentistry and Endodontics, Saveetha Dental college, Saveetha University, India)
}

\begin{abstract}
An endodontic Flare-up is defined as an acute exacerbation of asymptomatic pulpal or periapical pathoses after the initiation or continuation of root canal treatment.Pain may be seen after initiating endodontic treatment for a previously asymptomatic tooth or during the course of treatment or after obturation. Establishing the cause of the flare-up is important to initiate the proper treatment. In the following article, the possible etiological factors and their mechanisms have been explored and discussed.
\end{abstract}

Keywords: Acute, endodontics, Exacerbation, flare-up, biofilm.

\section{Introduction:}

An endodontic flare-up is a complication of endodontic treatment which is defined as an acute exacerbation of aymptomatic pulpal or periapical pathoses after the initiation or continuation of root canal treatment. Postoperative pain after endodontic treatment is an undesirable yet very common occurrence. Even with strict precautions taken, people still experience varying degrees of residual pain or even exaggerated responses during and following root canal treatment. Flare-ups are frequent complications which are disturbing to both patients and clinicians and are the cause of majority of endodontic emergencies resulting in unscheduled visits for treatment. A flare-up is characterized by pain and/or swelling that may arise following initial debridement of the root canals or even after obturation. The severity of a flare-up varies according to the severity of the patient's pre-operative pathosis, signs and symptoms. Flare-ups can also be caused by inadequate debridement and disinfection of the root canals, microbial persistence after instrumentation, overinstrumentation, irrigant extrusion or apical extrusion of infectious debris during instrumentation. Flare-ups can also be related to a variety of other causes. The prevalence of post-operative pain in numerous studies done in different settings have shown rates as low as $0.39 \%[1]$ and rates as high as $20 \%[2]$. The incidence of postoperative pain following endodontic treatment was reported to be from $3 \%$ to $58 \%$ [3]. The occurrence of flareups will have a significant impact on the patients and the clinicians. A thorough understanding of the etiology of flare-ups is required to prevent, properly diagnose and effectively manage patients presenting with pain or swelling.

The various factors that play a role in the incidence of flare-ups and in endodontic practice and their mechanisms are discussed below.

The etiology of flare-up is multifactorial. They include,

- Microbial factors

- Host factors - Patient's age, gender, stress and psychological factors, immunological status, systemic diseases, Local adaptation, periapical tissue response, Changes in periapical tissue pressure, effects of chemical mediators.

- Treatment procedures ( mechanical or chemical injuries) - Incomplete debridement, apical extrusion of debris, overinstrumentation, irrigant extrusion, overextended or underextended obturation.

\section{Microbial Causes:}

The most important goal of root canal therapy is to minimize the number of microorganisms and pathologic debris in root canal systems to prevent or treat apical periodontitis [4]. Many teeth present with infection and pain after endodontic intervension because of persisitent bacterial presence. Microorganisms play an important role in periradicular inflammation, both preoperatively and postoperatively. The mechanisms by which microorganisms cause flare-ups are many.

Failure of endodontic treatment and flare-ups can be attributed to microbial causes only if they are pathogenic, have sufficient numbers and have access to the periradicular tissues. The root canal system has an environment conducive for the survival of certain species of microorganisms. This environment is disrupted by endodontic treatment, by measures for disinfection, debridement, and intracnal medicaments. To survive in this altered environment where nutrient levels are low, bacteria must possess abilities to adapt themselves to the changed environment[5].

Bacteria residing in certain anatomical areas of the root canals such as isthmuses, apical ramifications, irregularities, deltas and dentinal tubules may be left untouched and unaffected by disinfection procedures $[6,7]$. 
These buried bacteria, after the canals are sealed, are killed or prevented from gaining access to the periradicular tissues by the root canal filling. Some bacteria can also remain viable by deriving nutrients from tissue remnants. An inadequate seal provided by the root canal filling can allow seepage of tissue fluids and provide a substrate for bacterial growth. The bacteria can also gain access to the periradicular area and cause inflammation[5].Many endodontic pathogens show an increased virulence when in association with other pathogenic species[8].

\subsection{Mechanisms For Survival:}

The bacteria have many mechanisms to withstand depletion of nutrients. In case of nitrogen depletion, bacteria that require ammonia as a source of nitrogen are able to scavenge traces of ammonia. This is enables by the Ntr gene system[9]. In situations of oxygen depletion, the Arc system (Aerobic respiration regulatory) is activated which helps the bacteria shift from aerobic to anaerobic metabolism[9]. Under low glucose concentrations, bacteria can activate catabolite repressor system which enables production of enzymes from other sources[9]. Under phosphate starvation, cells utilize organic phosphate compounds and scavenge trace amounts of inorganic phosphate[9].

\subsection{Bacterial Populations In The Root Canal:}

The microbiota in flare-ups and refractory or failed cases are different from untreated cases , the former having more of gram negative, facultatives and anaerobes and latter having more gram positive bacteria. The oxygen tension and oxidation-reduction potential are higher in coronal portion of the canals, thus housing facultatives and aero-tolerant anaerobes. Anaerobes are significantly higher in the apical third of the root canal due to the anaerobic conditions of the area [10].

\subsection{Sudden Change in Environment - Change in virulence:}

Chemo-mechanical preparation of the canal must be completed in one appointment and in intracanal medicament must be left in place and sealed until the next appointment. Incomplete preparation will only remove some of the pathogenic organisms leaving behind other bacteria that will multiply[11]. Changes in the environment can make previously inhibited bacteria to become virulent in cases where incomplete chemomechanical preparation is done[12]. When these organisms overgrow, and are virulent, they can cause periradicular tissue damage and exacerbation of symptoms.

Secondary infection of the root canal system can occur by organisms that were not initially present during the primary infection. Introduction of new organisms into the root canal can occur by contamination of irrigants, endodontic instruments [13]. Microorganisms can also enter through temporary restorative materials and obturated root canals when there is inadequate seal, fracture of tooth structure or a delay in placing a permanent restoration[14].

Matusow et al proposed that a change in oxidation-reduction potential of a root canal environment can be a cause for exacerbation of symptoms after endodontic treatment [15]. When the tooth is opened, oxygen enters the root canal system and the microbial population changes from anaerobic to aerobic. In an aerobic environment, the energy yield and growth rate are faster.

\subsection{Bacteroides Melaninogenicus:}

In a study done by Sundqvist et al in necrotic dental pulps, a relationship was established between certain microorganisms and painful teeth. After utilizing anaerobic techniques to identify these organisms, most of the strains were found to be obligately anaerobic. In all cases of flare-up, an anaerobic gram negative rod, Bacteroides melaninogenicus was found [16]. Also, Grifee et al did a study to reveal that symptomless infected teeth did not contain this organism [17]. This organism has been found to produce collagenolytic, fibrinolytic enzymes which activate Hageman factor to produce bradykinin which is a potent pain mediator.

Sudqvist also found combinations of bacteria that were responsible for transmissible infections, one such combination was B.melaninogenicus and Bacteroides asaccharolyticus. This showed the bacterial synergism that maintains bacteroides infections [18].

\subsection{Fusobacterium nucleatum:}

A study was done to determine the association of Fusobacterium nucleatum and endodontic flare-ups. The cases were pre-operatively asymptomatic with periapical lesions. Root canal samples were taken after the occurrence of flare-ups and the bacterial findings were correlated with the pain intensity recorded on a visual analog scale. All teeth in patients who reported with severe pain and swelling had Fusobacterium nucleatum. Samples from teeth with less pain did not have F.nucleatum. Thus, F.nucleatum appeared to be associated with development of the most severe forms of flare-ups. Bacteria that were also frequently recovered from the samples were Prevotella species and Porphyromonas species [19]. 
Gram positive organisms can also be involved in flare-up. Teichoic acids which are part of their cell walls can act as potent immunogens and induce inflammation [17].

\subsection{Enterococcus Faecalis:}

Enterococcus faecalis is a persistent organism that makes up a small portion of the endodontic microflora but has a major role in the etiology of persistent periradicular infection[20]. These are facultative anaerobes that can survive in harsh environments including extremely alkaline $\mathrm{pH}$. Studies have shown that E.faecalis is present in 4 to $40 \%$ of primay endodontic infections. The likeliness of its presence in failed root canal treatment was found to be nine times higher. E.faecalis has been detected at $67-77 \%$ in a PCR detection method[21]. It is known to be resistant to the action of calcium hydroxide intracanal medicament. It can survive in the presence of calcium hydroxide due to the presence of a proton pump and its ability to passively maintain $\mathrm{pH}$ homeostasis.

\subsection{Actinomyces radicidentis:}

A new bacterial species has been identified with failed endodontic treatment by Kalfas et al. In two patients with failed endodontic treatment and persistent signs and symptoms, the root fillings were removed and the samples were grown in pure culture. The bacteria were similar to each other and were classified as Actinomyces radicidentis[22].

\subsection{Biofilm in endodontic infections:}

A biofilm is a group of adherent microorganisms embedded in a matrix of extracellular polymeric substance, which forms an intermicrobial matrix. Within a biofilm, the bacteria are more protected and show a higher resistance to antimicrobial agents and host defence mechanisms. Tornstad et al reported the presence of biofilms near the apical foramen and bacterial colonies inside periradicular granulomas[22]. A study was done to examine electron microscopically the surface of extracted teeth, root tips and gutta percha points for the presence of biofilm formation in periapical lesions. Similar biofilm structures were found on the extracted teeth and the gutta percha pointsand in the extraradicular structures within the body of the lesion. This biofilm was shown to be associated with refractory periodontitis [23].

Quorum sensing a bacterial cell to cell signaling mechanism that helps in communication between the organisms in a biofilm. This is mediated through signaling molecules that when present above a threshold amount, can induce changes in the gene expression of other organisms. This helps in regulation of several properties like virulence, ability to form biofilms, incorporate extracellular DNA and coping with change in environment [24]. Many of the oral bacteria found in the root canals like Streptococcus mitis, S.gordonii, P.gingivalis, Prevotella intermedia, Fusobacterium nucleatum have the ability to communicate with each other in a biofilm. When there is a change in the environment, or in harsh environments, these signal molecules alter gene expression to modify the phenotypic properties of the biofilm bacteria and make them resistant to the treatment. Flare-up lesions have the same mechanism. A shift in the flow of nutrients when a tooth is opened up, causes ecological changes and promotes growth of proteolytic bacteria. This causes acute exacerbations. Overinstrumentation may cause entry of inflammatory exudates into the root canal and cause growth of proteolytic bacteria [25].

\section{Host Factors:}

Different individuals present with different patterns of resistance to pulpal and periradicular disease and endodontic treatment. There are many host factors that might play a role in the incidence of flare-ups in patients.

\subsection{Age \& Gender:}

A longitudinal study was done to assess the prevalence of post-obturation pain in patients undergoing root canal treatment and to evaluate the influence of factors affecting pain. $40.2 \%$ of patients reported with pain after treatment. The patient's gender was found to be a prognostic determinant. Females were found to have an increased rate of flare-ups than males[26]. Age did not have any significant influence on the incidence of postobturation pain. There are possible explanations for this increased prevalence among females as proposed by Marcus in 1995 and Fillingim \& Maixner 1995[27,28]. Other determinants included tooth type, presence of periapical lesions, history of post-operative pain and swelling and single or multiple visit treatment. Fluctuating female hormone levels cause an increased level of serotonin and noradrenaline leading to increased pain prevalence during the menstrual period and in women receiving hormonal replacement therapy or oral contraceptives. Another study was done to determine pain experienced by patients during endodontic treatment and to correlate this to age, gender, diagnosis, periapical pathosis, etc. Root canal treatment with irreversisble pulpitis and acute apical periodontitis was more painful. Women felt a slightly higher percentage of pain $(42 \%)$ 
than men(26\%). $60 \%$ of patients who were 35 or younger experienced some type of pain, whereas only $39 \%$ of patients older than 35 experienced pain [29].

\subsection{Psychological factors and anxiety:}

Fear of dentists, dental procedures, anxiety and other psychological factors influence a patient's risk of developing pain and their pain perception[16]. Previous traumatic dental experiences may also play a role in flare-up rate. These anxieties can initiate and aggravate or intensify pain.

\subsection{Periapical Tissue response:}

Selye introduced the concept of alteration in local adaptation as a potential cause for flare-ups. When a chronic inflammation exists, the periapical tissue is adapted to the irritant. This is the reason why the inflammation may exist without causing much pain or swelling. But when endodontic treatment is initiated, new irritants in the form of irrigants, instruments, intracanal medicaments are introduced into the periapical area that may lead to a violent reaction causing severe pain and swelling[17]. The flare-up is also caused by introduction of salivary products, or forcing of microorganisms into a previously adapted environment[17].

Mohorn et al indicated the presence of changes in tissue pressure due to endodontic intervention. A positive or increased apical pressure showed the presence of exudates that has not been absorbed by the lymphatics and wiil cause pain due to pressure on nerve endings. This type of lesions will drain when the tooth is opened. In teeth with reduced or negative tissue pressure, microorganism can be aspirated into the periapical area resulting in the aggravation of the inflammatory response and pain.[30].

\subsection{Immunological phenomena:}

Chemical mediators like histamine, serotonin, prostaglandins, leukotrienes, etc play a role in the causation of pain. Histamine and serotonin, when released as a result of inflammation act on the blood vessels and increase vascular permeability. Prostaglandins, found in exudates increase vascular permeability, promote chemotaxis, induce fever, and sensitize pain receptors to stimulation by other chemical mediators[17]. Other plasma-derived factors include Hageman factor, Bradykinin, plasmin, etc. Plasmin activates Hageman factor, which in turn activates pre-kallikrein. Pre-kallikrein is in turn activated by plasmin to form Kallikrein. Kallikrein cleaves kininogen to form bradykinin. Bradykinin is a known pain mediator[17].

Cyclic nucleotides such as cyclic AMP and Cyclic GMP also influence pain transmission. Increased cyclic AMP levels reduce transmission of nerve impulses through hyperpolarization [31]. Another cyclic nucleotide, cyclic GMP has effects opposite that of cyclic AMP. Cyclic GMP enhances depolarization and pain transmission[32]. Investigations have shown that there is a relative increase in cyclic GMP over cyclic AMP in cases of painful teeth[33].

When root canal treatment is initiated, instrumentation initiates an acute inflammatory process. As a result, there is an intense polymorphonuclear neutrophil infiltrate which release lysosomal enzymes which are capable of causing damage to the tissue, finally producing severe pain and swelling[17].

The pulp is known to have the capacity to produce antibodies to various antigens. Macrophages and plasma cells are present in pulpitis and periapical pathosis, which are involved in humoral immunity. Immunoglobulins have been detected in many periradicular lesions including granulomas and radicular cysts [34]. In addition to bacterial antigens, when treatment is initiated, medicament altered tissue, irrigants, sealers and root-canal filling materials have also been reported to induce immunological reactions[35].

\subsection{Systemic diseases:}

Presence of systemic diseases also contribute to the severity of endodontic infections and the response to treatment. In a study of outcome of endodontic treatment in diabetic and non-diabetic patients, in patients diagnosed with periradicular lesions, diabetics on insulin tended to have an increased incidence of periradicular pain compared to non-diabetics. They also had twice the rate of flare-ups compared to non-diabetics[36].

\section{Treatment Factors:}

The main purpose of biomechanical preparartion is to clean the root canals and disinfection, to rid it of microorganisms that will cause persistent infection. Inadequate preparation can cause acute exacerbations [37]. Apical preparation of the root canals during chemo-mechanical preparation is a critical step and an essential part of the cleaning and shaping. Shuping et al and Siqueira et al demonstrated that larger apical instrumentation sizes are needed to allow irrigating solution to reach the apex [38,39]. Failing to clean canals, especially in the apical region, can result in treatment failure. 


\subsection{Inadequate Debridement:}

A study was done to correlate the presence of bacterial infection in the canal system and the presence of periradicular radioluscencies with the occurrence of endodontic flare-ups. They did a biopsy study of the teeth with flare-ups and found that part of the root canal space was left untouched during chemomechanical debridement. Therefore, teeth with pre-operative periradicular radioluscencies fail more frequently after root canal treatment[30].

\subsection{Intracanal medicaments and obturating materials as antigens:}

Intracanal medicaments are used in the root canals for their anti-microbial effects and to reduce the incidence of flare-ups. But, allergic responses to components of intracanal medicaments, irrigants and sealers have been studied. The medicaments can act as antigens and produce exaggerated responses and cause pain. Some chemicals used as intarcanal medicaments and irrigants like sodium hypochlorite, hydrogen peroxide, eugenol, iodine compounds, prarchlorophenol, formocresol can act as antigens and induce a hypersensitivity response[37]. This can be explained by a sudden imbalance in the root canals when the agent comes in contact with the periapical tissues when it is introduced deep into the root canals, or when there is overfilling[37].

In a study done to investigate the relationship between postoperative pain and the use of various intracanal medicaments, the patients were given Calcium hydroxide dressings and some patients were given Ledermix paste dressing (corticosteroid-antibiotic paste). Patients with corticosteroid dressing experienced less postoperative pain when compared with calcium hydroxide and those with no dressing [40].

\subsection{Irrigant extrusion:}

Irrigation is an important step during chemo-mechanical preparation. Sodium hypochlorite and hydrogen peroxide are two of the commonly used intracanal irrigants. Sodium hypochlorite has excellent antimicrobial efficiency, and ability to dissolve necrotic as well as vital tissue [41]. Inadvertant extrusion of irrigants beyond the apical foramen will lead to violent reactions - pain, swelling, haematoma, burning sensation, ulceration, tissue necrosis. Also, excessive pressure during irrigation will cause large amounts of irrigant to come in contact with the periapical tissues [42]. Use of hydrogen peroxide as irrigant has also caused adverse reactions like sudden, severe pain along with rapid swelling and erythema in the region [43].

\section{Conclusion:}

Flare-ups are an undesirable occurrence in the course of endodontic treatment that causes distress to both the patient and operator. Clinicians should employ appropriate measures in an attempt to prevent the occurrence of flare-ups and should be able to treat the efficiently when they do occur.

\section{References:}

[1] Iqbal M, Kurtz E, Kohli M, Incidence and factors related to flare-ups in a graduate endodontic programme, International Endodontic Journal, (42), 2009, 99-104.

[2] Morse DR, Koren LZ, Esposito JV, Goldberg JM, Belott RM, Sinai IH et al, Asymptomatic teeth with necrotic pulps and associated periapical radioluscencies: relationship of flare-ups to endodontic instrumentation, antibiotic usage and stress in three different time periods, International journal of Psychosomatic research, (33), 1986, 5-87.

[3] Sathorn C, Parashos P, Messer H, The prevalence of post-operative pain and flare-up in single- and multiple- visit endodontic treatment : A systematic review, Internayional Endodontic Journal, (41), 2008, 91-99.

[4] Dean Baugh, James Wallace, The role of apical instrumentation in root canal treatment: A review of the literature, Journal of Endodontology, 31(5), 2005, 333-340.

[5] J.F.Siqueira Jr, Aetiology of root canal treatment failure: why well-treated teeth can fail, International endodontic journal, (34), 2001, 1-10.

[6] Lin LM, Pascon EA, Skribner J, Gaengler P, Langeland K. 1991, Clinical, radiographic and histopathological study of endodontic treatment failures, Oral surg oral medicine oral pathology oral radiology and endodontics, (71), 603-611.

[7] Siqueira JF Jr, Uzeda M, Fonseca MEL. 1996, Scanning electron microscopic evaluation of in vitro dentinal tubules penetration by selected anaerobic bacteria, Journal of Endodontology, (22), 308-10.

[8] Atlas RM, Principles of microbiology. $2^{\text {nd }}$ edition, 1977, Dubuque, IA, USA, WCB Publishers.

[9] Fabricius L, Dahlen G, Ohman AE, Moller AJR, predominant indigenous oral bacteria isolated from infected root canals after varied times of closure, Scandinavian journal of dental research, (90), 1982, 134-144.

[10] J.F.Siqueira Jr, microbial causes of endodontic flare-ups, International endodontic journal, (36), 2003, 453-463.

[11] Sundqvist G, Ecology of the root canal flora, Journal of endodontics,(18), 1982, 427-430.

[12] Siqueira JF Jr, Lima KC, Staphylococcus epidermidis and Staphylococcus xylosus in a secondary root canal infection with persistent symptoms: a case report, Australian endodontic journal ,(28), 2002, 61-63.

[13] Siqueira JF Jr, Rocas IN, Favieri.A, Abad EC, Castro AJR, Gahvya SMM, Bacterial leakage in coronally unsealed root canals obturated with three different techniques, Oral surg oral medicine, oral pathology, oral radiology endodontics, (90), 2000, 587590.

[14] Matusow RJ, Endodontic cellulitis' flare-up' Case report, Australian dental journal, (40), 36-38.

[15] Siqueira JF Jr., Magalhaes FA, Lima KC, Uzeda M, Pathogenecity of facultative and obligate anaerobic bacteria in monoculture and combined with either Prevotellq intermedia or Prevotella nigrescens, Oral microbiology and immunology,(13), 1998, 368-372.

[16] Selye H, The part of inflammation in the local adaptation syndrome. In: Jasmin G, Robert A, eds. The mechanism of inflammation, Acta Montreal, 1953. 53-74. 
[17] Samuel Seltzer, Irving J.Naidorf, Flare-ups in endodontics: I. Etiological factors, Journal of endodontics, 30(7), 2004, 476-481.

[18] Sundqvist G, Bacteriological studies of necrotic dental pulps (dissertation) Umea, Sweden. 1976. 24-27.

[19] Luis Eduardo Chavez de Paz Villanueva, Fusobacterium nucleatum in endodontic flare-ups, Oral surgery oral medicine oral pathology oral radiology and endodontology, 93(2), 2002, 179-183.

[20] Charles H.Stuart, Scott A.Schwartz, Thomas J.Beeson, Christopher Owatz, Enterococcus faecalis: its role in root canal treatment failure and current concepts in retreatment, Journal of endodontology, (32), 2006, 93-98.

[21] Rocas IN, Siqueira JF, Santos KRN, Association of Enterococcus faecalis with different forms of periradicular diseases, Journal of endodontology, (30), 2004, 315-320.

[22] Grifee M, Patterson SS, Miller CH, Kafawry AH, Newton GW, The relationship of Bacteroides melaninogenicus to symptoms associated with pulpal necrosis, Oral surgery, (50), 1980, 457-461.

[23] Louis.M.Lin, Joseph E.Skribner, Peter Gaengler, Factors associated with endodontic treatment failure, Journal of endodontics, 18(12), 1992, 625-627.

[24] Cvitkovitch DG, Li Y-H, Ellen R, Quorum sensing and biofilm formation in Streptococcal infections, Journal of Clinical Investigation, (112), 2003, 1626-1632.

[25] Gunnel Svensater, Gunnar Bergenholtz, Biofilms in Endodontic infections, Endodontic topics,(9), 2004, 27-36.

[26] Y.-L. Ng, J.P.Glennon, D.J. Setchell, K.Gulabivala, prevalence of and factors affecting post-obturation pain in patients undergoing root canal treatment, International endodontic journal, (37) , 2004, 1-391.

[27] Marcus DA.1995, Interrelationships of neuro-chemicals, estrogen and recurring headache Pain, (26), 129-139.

[28] Fillingham RB, Maixner W, Gender difference in the response to noxious stimuli, Pain Forum, (4), 1995, 209-221.

[29] J.J.Segura-Egea, R.Cisneros-Cabello, J.M. Llamas-Carreras, E.Velasco-Ortega, Pain associated with root canal treatment, International Endodontic journal, (42), 2009, 614-620.

[30] Mohorn HW, Dowson J, Blankenship JR, Odontic periapical pressure following vital pulp extirpation, Oral Surgery, (31), 1971, 536 .

[31] Greengard P, Possible role for cyclic nucleotides and phosphorylated membrane proteins in post-synaptic actions of neurotransmitters, Nature, 260:101, 1976.

[32] Morrison DC, Henson PM, Release of mediators from mast cells and basophils induced by different stimuli. In: Bach MK, ed. Immediate hypersensitivity, Immunology series, New York: Marcel Deckker, 7, 1978, 431-502.

[33] Sproles AC, Schilder H, Schaffer LD, Cyclic AMP and cyclic GMP concentrations in normal and pulpitic human dental pulps, Journal of Dental Research, 58:2369, 1979.

[34] Kuntz DD, Genco RJ.Localization of immunoglobins and complement in persistent periapical lesions, Journal of Dental Research, 53:215, 1974 .

[35] Block RM, Lewis RD, Sheats JB, Burke SH, Fawley J, Antibody formation and cell-mediated immunity to dog pulp tissue altered by eight endodontic sealers via the root canal, International Endodontic Journal, 15:105, 1982.

[36] Fouad AF, Burleson J, The effect of diabetes mellitus on endodontic treatment outcome: data from an electronic patient record, Journal Am Dental Association, (134), 2003, 43-51.

[37] A.Rimmer, Intracanal medications and antibiotics in the control of inter-appointment flare-ups, Quintessence international, 22(12), 1991, 997-1005.

[38] Shuping G, Orstavik D, Sigurdsson A, Trope m, Reduction of intracanal bacteria using nickel-titanium rotary instrumentation and various medications, Journal of Endodontolgy, (26), 2000, 751-755.

[39] Siqueira J, lima k, Magalhaes F, Lopes H, de Uzeda M, Mechanical reduction of the bacterial population in the root canal by three instrumentation techniques, Journal of Endodontology, (25), 322-325.

[40] E.H.Ehrmann, H.H.Messer, G.G.Adams, The relationship of intracanal medicaments to postoperative pain in endodontics, International endodontic journal, (36), 2003, 868-875

[41] Senia ES, Marraro RV, Mitchell JL 1975, Rapid sterilization of gutta-percha cones with 5.25 per cent Sodium hypochlorite and hydrogen peroxide versus normal saline solution, Journal of Endodontics, (1), 136-140.

[42] M.Hulsmann, W.Hahn, Complications during root canal irrigation - literature review and case reports, International endodontic journal,(33), 2000, 186-193.

[43] Patterson CJ, McLundie AC. 1989. Apical penetration by a root canal irrigant: a case report, International Endodontic journal, (22), 197-199. 